Supporting Information (SI) for

\title{
2 Alginate Biopolymer Effect on the Electrodeposition of Manganese Dioxide Electrode for Supercapacitors
}

Kethaki Wickramaarachchi, Manickam Minakshi Sundaram*, David J. Henry, and Xiangpeng Gao College of Science, Health, Engineering and Education, Murdoch University, WA 6150, Australia

*E: m.minakshi-sundaram@murdoch.edu.au ; T.+61 893602017

Brief description: Additional information covering both physicochemical and electrochemical concentrations of alginate biopolymer additive, along with equations to calculate the energy storage, structure, BET surface area and CV analysis results.

\section{Contents}

Equations.

Figure S1....

Table S1....

Figure $\mathrm{S} 2$.

Figure S3

Table S2.

Figure S4 ...

S8

23

Figure S5.

Figure S6. 


$$
C_{s, C V}=\int_{0}^{0.6} \frac{i d v}{2 V_{s} m \Delta v}=\frac{Q}{m \Delta v}
$$

30

31

$$
C_{S, C D}=\frac{I \Delta t}{m \Delta v}
$$

2

33

34

$$
E=\frac{1}{2} C_{s, C D} \frac{\Delta v^{2}}{3.6}
$$

35 where $i$ - current (A), $V_{S}$ - scan rate $\left(\mathrm{V} \mathrm{s}^{-1}\right), Q$ - Average charge during a cathodic anodic scan (C), 36 37

38

39

40

41

$$
P=\frac{E}{\Delta t} \times 3600
$$
$I$ - current rate (A), $\Delta t$ - time for discharge (s), $\Delta v$ - potential window (V), $m$ - active electrode $\operatorname{mass}(\mathrm{g})$.

$$
\frac{m^{+}}{m^{-}}=\frac{C^{-} \cdot \Delta E_{m}^{-}}{C^{+} \cdot \Delta E_{m}^{+}}
$$

where, $C^{+}, m^{+}, \Delta E_{m}^{+}$and $C^{-}, m^{-}, \Delta E_{m}^{-}$are specific capacitance, mass, safe operation potential window of the positive and the negative electrodes. The electrode mass ratio determined as 0.30 .

42

43

44

45

46

47

48

49

50

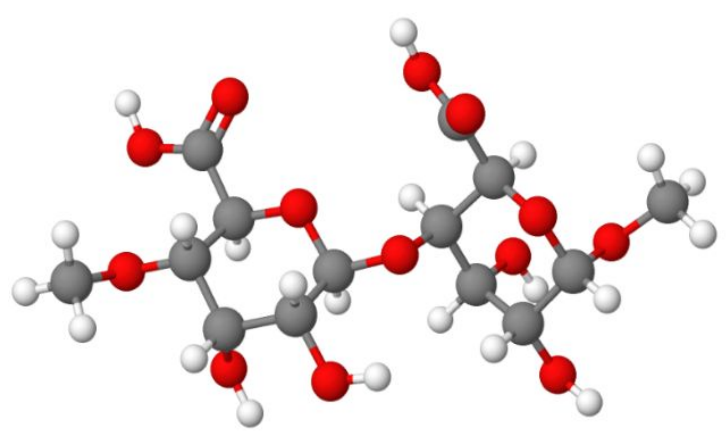

- Hydrogen - Carbon - Oxygen

Figure S1 Chemical structure of the Alginic acid polymer. 


\section{Surface area and pore distribution}

53 Nitrogen adsorption-desorption analyses were performed to examine the specific surface area, total pore volume, and pore diameter of the pristine EMD and EMD/Alg composites. EMD flakes were ground into powder and degassed before the BET analysis. The Brunauer-Emmett-Teller (BET) analyses of pristine EMD and EMD/Alg composites in Figure S2 show a type IV isotherm with an $\mathrm{H} 2$ hysteresis loop in the range $0.47-0.8 \mathrm{P} / \mathrm{P}_{\mathrm{o}}$ according to the IUPAC classification. ${ }^{1}$ The measured average pore sizes and pore volumes are presented in Table S1. The results indicate that the addition of Alg biopolymer has improved the surface area, and the extent being larger for the optimized amount of Alg, which is EMD/0.5 Alg exhibiting $97.1 \mathrm{~m}^{2} \mathrm{~g}^{-1}$ with an average pore diameter of $3.84 \mathrm{~nm}$. The BET surface areas for electrodeposited EMD reported in the literature were in the range of $10-100 \mathrm{~m}^{2} \mathrm{~g}^{-1}$.,3 The hysteresis loop at relative pressure closer to unity in the adsorption-desorption curves indicates the presence of mesopores in the material. ${ }^{4} \mathrm{~A}$ significant increment of pore volume (from $0.1 \mathrm{~cm}^{3} \mathrm{~g}^{-1}$ to $0.7 \mathrm{~cm}^{3} \mathrm{~g}^{-1}$ ) in the particle diameter ranging from 2 to $6 \mathrm{~nm}$ has been observed from the curves obtained by Barret-Joyner-Halenda (BJH) method for EMD/0.5Alg composite as illustrated in Figure S2-D.

67 68

Table S1 Surface area and pore size distribution values for the pristine EMD and EMD/Alg composites.

\begin{tabular}{|c|c|c|c|}
\hline Sample & $\begin{array}{c}\text { BET surface } \\
\text { area }\left(\mathbf{m}^{\mathbf{2}} \mathbf{g}^{-\mathbf{1}}\right)\end{array}$ & $\begin{array}{c}\text { BJH adsorption } \\
\text { average pore } \\
\text { diameter } \mathbf{( n m})\end{array}$ & $\begin{array}{c}\text { BJH Adsorption } \\
\text { cumulative pore } \\
\text { volume }\left(\mathbf{c m}^{\mathbf{3}} \mathbf{g}^{-\mathbf{1}}\right)\end{array}$ \\
\hline EMD & 80.4 & 4.57 & 0.08 \\
\hline EMD/ 0.1 Alg & 83.2 & 4.00 & 0.08 \\
\hline EMD/ 0.25 Alg & 84.8 & 4.13 & 0.07 \\
\hline EMD/ 0.5 Alg & 97.1 & 3.84 & 0.08 \\
\hline EMD/ 1 Alg & 94.6 & 4.15 & 0.08 \\
\hline
\end{tabular}



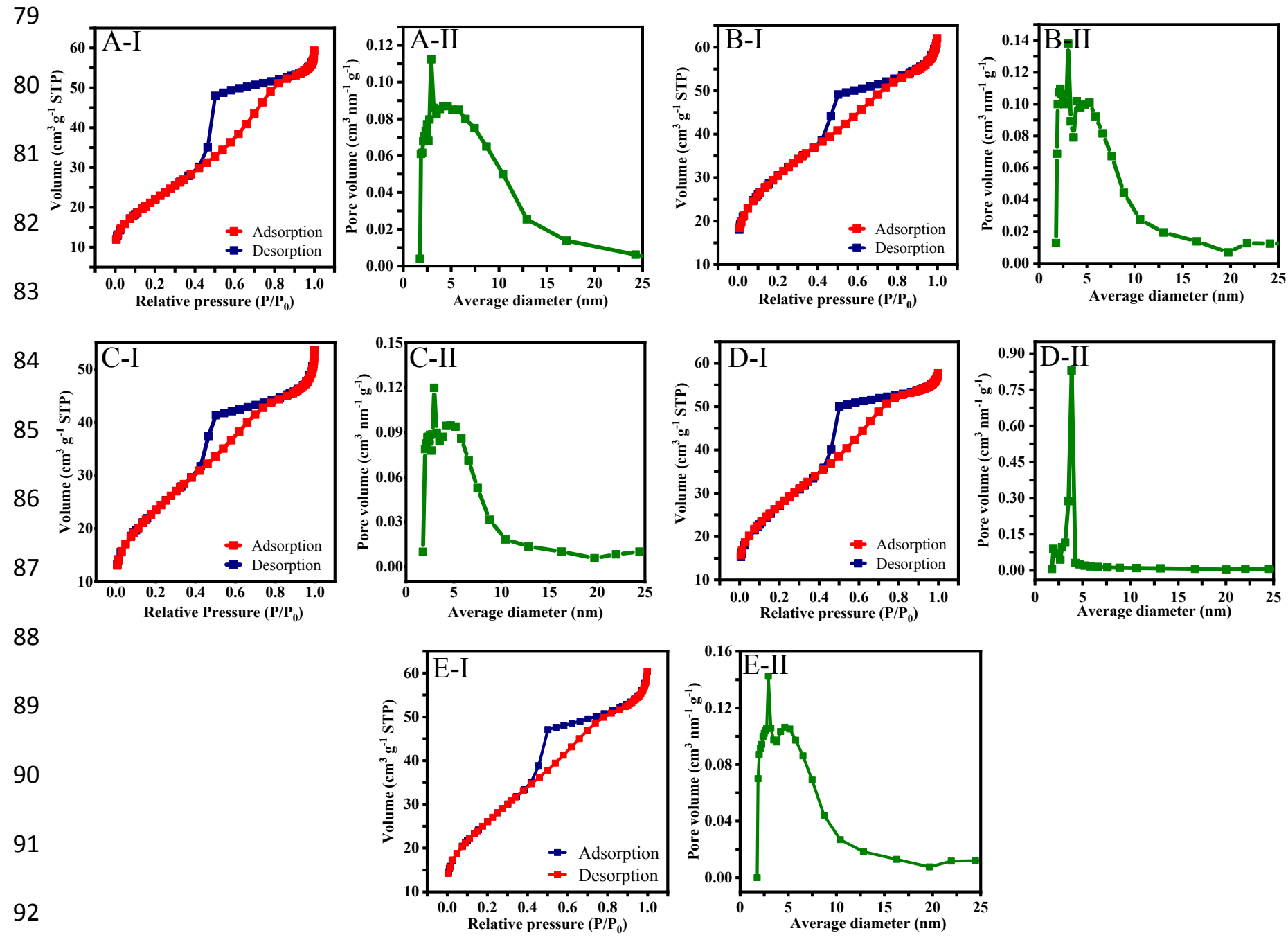

93 Figure S2 Nitrogen adsorption and desorption isotherms of (A-I, A-II) Pristine EMD, (B-I, B-II) EMD/0.1 Alg, (C-I, C-II) EMD/0.25 Alg, (D-I, D-II) EMD/0.5 Alg, (E-I, E-II) EMD/1 Alg. 


\section{Electrochemical characterization of the single electrode system}

102 Capacitive performance of EMD/Alg composites

103 The CV curves in Figure S3 (A-I, B-I, C-I, D-I, E-I) show increased current response area under 104 the curve for the EMD/Alg composites compared to that of the pristine EMD, thereby establishing 105 beneficial charge storage properties. The current response increases with the increase in the concentration of Alg in the bath, but after the threshold ( $\geq 0.5 \mathrm{Alg}$ ), for $1 \mathrm{Alg}$, the current response decreases. Despite these, for all the biopolymer Alg concentrations, the CV curves invariably show cathodic (reduction), and anodic (oxidation) peaks during the analyses. The quasi-rectangular shape of the obtained peaks indicates that the charge storage mechanism involves charge transfer corresponding to be pseudocapacitive. For the EMD/0.5 Alg (Figure S3 D-I), the shape of the CV curve resembles or approaches electrochemical double layer (EDLC) behavior. The corresponding quasi-triangular shaped charge-discharge curves are shown in Figure S3 (A-II, B-II, C-II, D-II, EII). The applied current rate $(0.5-3 \mathrm{~mA})$ is inversely proportional to the charge-discharge time, implying at a lower current rate of $0.5 \mathrm{~mA}$ accumulation of ions from the electrolyte with the electrode interface gets longer, resulting in a longer discharge time. For the EMD/Alg composites, the IR drop of the discharge cycle is negligible, indicating the polymer is beneficial in reducing the IR drop and electrode resistance; this is further confirmed by electrochemical impedance spectroscopy (EIS) analysis. Among the concentrations studied, the EMD/0.5 Alg in Figure S3 DII shows increased charge-discharge time, thereby indicating a better charge storage mechanism for the EMD/Alg composites. However, at a higher concentration of Alg (1 Alg), the chargedischarge time gets similar to that of pristine EMD, suggesting the extensive aggregation of the carboxylic group with Mn cation led to poor behavior. 

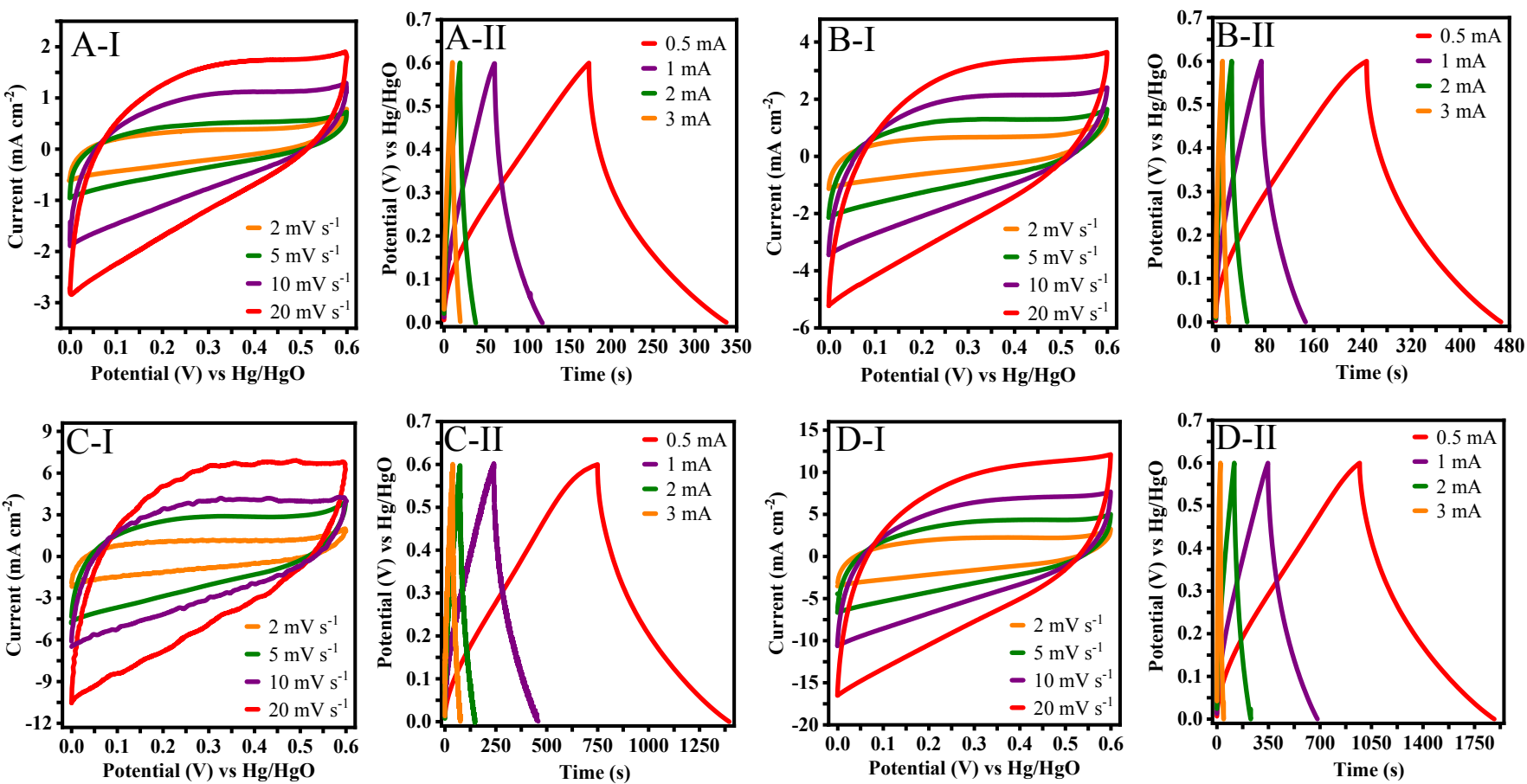

136

137

138

139

140
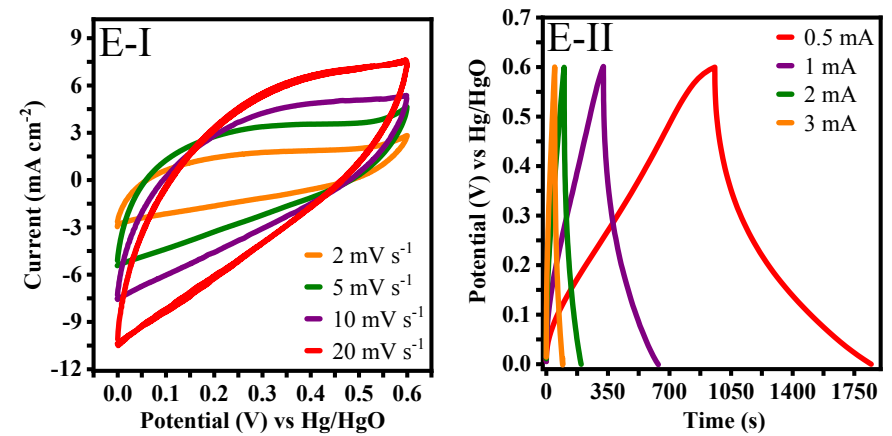

141

Figure S3 Cyclic voltammograms (CV) and galvanostatic charge-discharge (GCD) profiles of (AI, A-II) pristine EMD, (B-I, B-II) EMD/0.1 Alg, (C-I, C-II) EMD/0.25 Alg, (D-I, D-II) EMD/5

143 Alg, (E-I, E-II) EMD/1 Alg and the bio polymer-modified EMD (Note. the axes scales are different for CV and GCD curves of each sample). 


\section{Electrochemical impedance spectroscopy (EIS) studies}

The EIS measurements along with fitted curves (in Figure S4) closely agree with the experimental data collected before and after 1000 cycles in the three-electrode configuration. The equivalent circuit was built using EC-Lab Z fit. The difference observed between the initial and after 1000 cycles is not significant. Typically, the Nyquist plot consists of a semicircle at high frequency, followed by a straight line. In the EMD/0.5 Alg, Nyquist plot (in Figure S4-A) showed only a diffused semicircle at a high frequency that tails off linearly at lower frequencies. The charge transfer resistance $\left(\mathrm{R}_{\mathrm{ct}}\right)$, which is determined from the semicircle diameter, is shown in Table S2. The ionic charge at the interface contributes to the ideal double-layer capacitance. However, the coated electrode's surface roughness and porosity could produce overlapping double layers that caused deviations from the ideal capacitance. Therefore, the non-ideal capacitance is usually represented by a constant phase element ${ }^{5-8}$ (CPE1 in Figure S4-B), parallel to the charge transfer resistance $\mathrm{R}_{\mathrm{ct}}$.

During the charge transfer, when the protons and electrons are inserted into the EMD structure, a diffusion component should also be considered for charge diffusion. At higher frequencies, the diffusion of charge (insertion and extraction) will occur considerably faster; therefore, the impact given to the impedance by diffusion will not be significant. At lower frequencies, the inserted electrons and ions tend to penetrate more depth; hence the mass transfer by diffusion would be the limiting factor to determine the impedance. This phenomenon is accounted for by the Warburg circuit element (W in Figure S4-B). However, the structural disorder and porous surface of the crosslinked EMD will overrule the underlying assumption of Warburg impedance that applied only to planar and semi-infinite diffusion of charge. This was compensated by the second constant phase element (CPE2 in Figure S4-B) was included in the circuit in series with Warburg element and $\mathrm{R}_{\mathrm{ct}}$. The values of the parameters according to modified Randle's circuit are shown in Table S1. Based on the results, only minimal changes have occurred for both $R_{s}$ and $R_{c t}$. The ' $n$ ' is a number associated with the constant phase element CPE can vary between 0 to $1(0 \leq n \leq 1)$. If the ' $n$ ' value is equal to 0 , it shows the pure resistance, and if the value is 1 , it is pure capacitance. According to ' $n$ ' parameters given by the model, the ' $n_{1}$ ' has a moderate value of about 0.6 while the ' $\mathrm{n}_{2}$ ' is about 0.9 before and after charge-discharge cycling. The kinetic study concludes that 
179 180

186

187

188

189

190

191

192

193

194

195 196

197

198

199

crosslinked EMD seems to be stable for long-term cycling, and porosity enhances the ion transport for charge storage.

Table S2 Equivalent circuit parameters from the fitting using the modified Randles circuit.

\begin{tabular}{|c|c|c|c|c|c|c|c|}
\hline & $\begin{array}{l}\mathbf{R}_{\mathrm{s}} \\
(\mathbf{\Omega})\end{array}$ & $\begin{array}{l}\mathbf{R}_{\mathrm{ct}} \\
(\boldsymbol{\Omega})\end{array}$ & $\begin{array}{c}\sigma{ }^{*} \\
\left(\Omega s^{0.5}\right)\end{array}$ & $\begin{array}{c}\text { CPE } 1 \times 10^{3} \\
\left(F s^{n_{1}-1}\right)\end{array}$ & $\mathbf{n}_{1}$ & $\begin{array}{c}\text { CPE } 2 \times 10^{3} \\
\left(\mathrm{~F} \mathrm{~s}_{\mathbf{n}^{-1}}\right)\end{array}$ & $\mathbf{n}_{2}$ \\
\hline $\begin{array}{l}\text { Before } \\
\text { cycling }\end{array}$ & 9.5 & 11.5 & 4.2 & 6.2 & 0.69 & 0.035 & 0.99 \\
\hline $\begin{array}{l}\text { After } \\
\text { cycling }\end{array}$ & 9.8 & 10.1 & 4.4 & 5.9 & 0.67 & 0.030 & 0.94 \\
\hline
\end{tabular}

${ }^{*} \sigma$ is Warburg coefficient

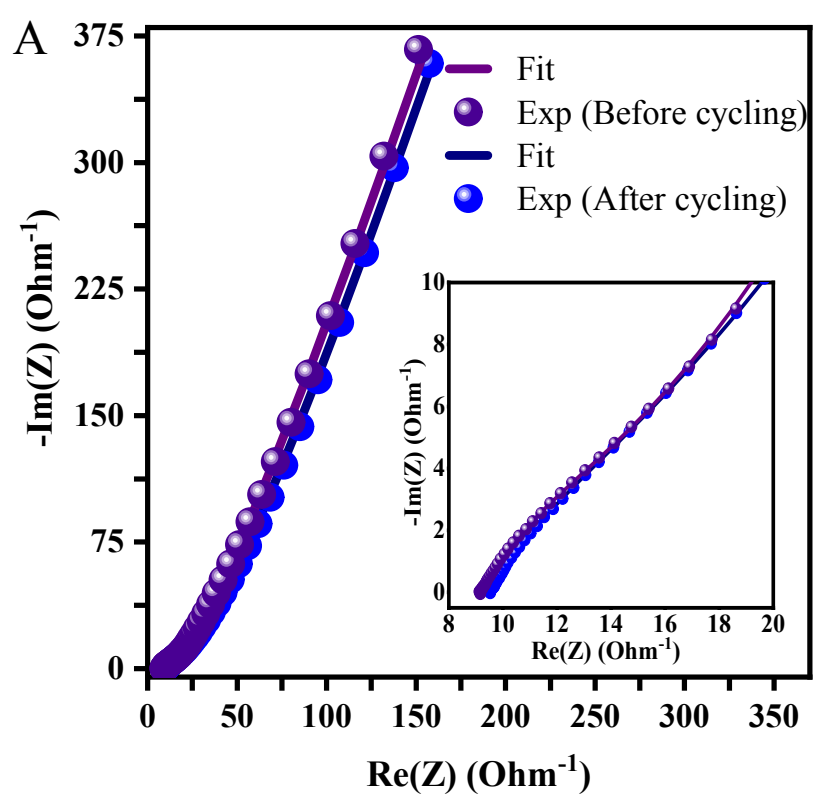

$\mathrm{B}$

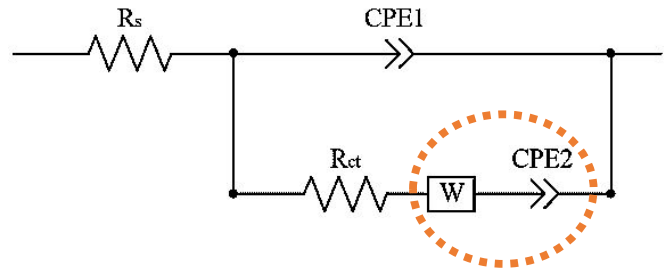

Figure S4 (A) Electrochemical impedance spectra of the EMD/0.5 Alg in $2 \mathrm{M} \mathrm{NaOH}$ electrolyte for single electrode cell (before and after 1000 cycles) and fitted curve, (B) Equivalent circuit model employed in EIS analysis. 

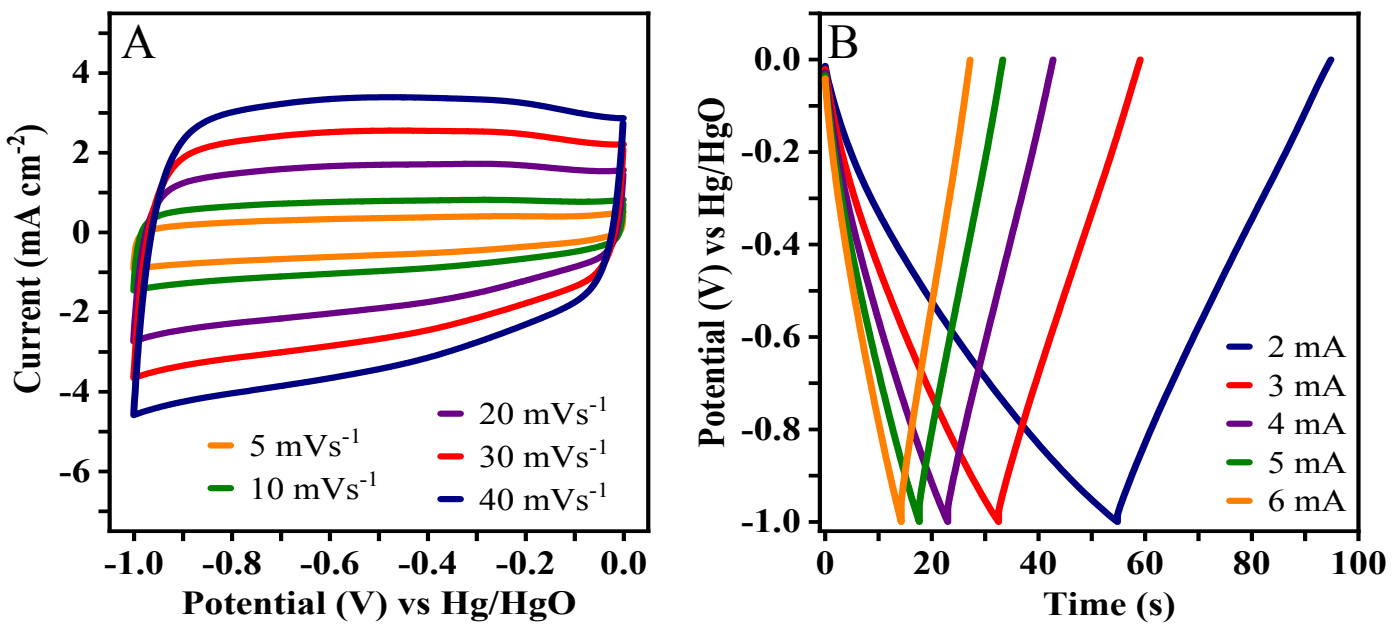

208

Figure S5 (A) Cyclic voltammetry and (B) Galvanostatic charge-discharge profiles of activated 209 carbon (anode) at different scan rates and current rates, respectively.

210

211

212

213

214

215

216

217

218

219

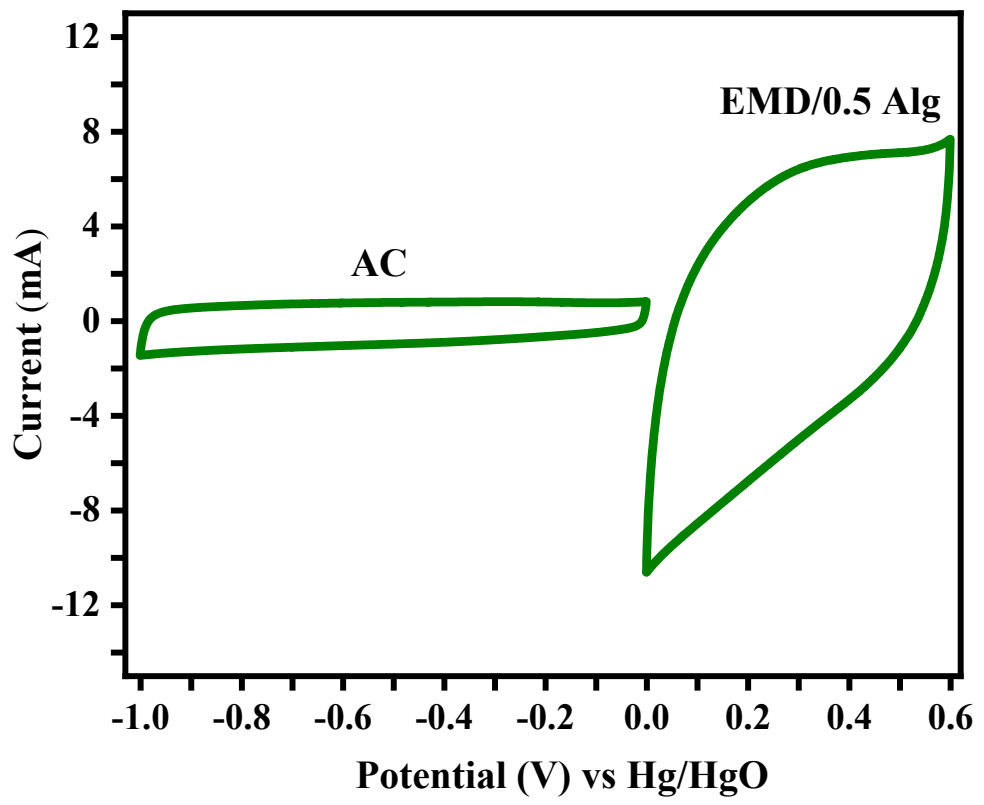

220

Figure S6 Cyclic Voltammetry profiles of Activated carbon (negative potential window -1 V), and $\mathrm{EMD} / 0.5 \mathrm{Alg}$ (positive potential window $0.6 \mathrm{~V}$ ) at $10 \mathrm{mV} \mathrm{s}^{-1}$ scan rate. 


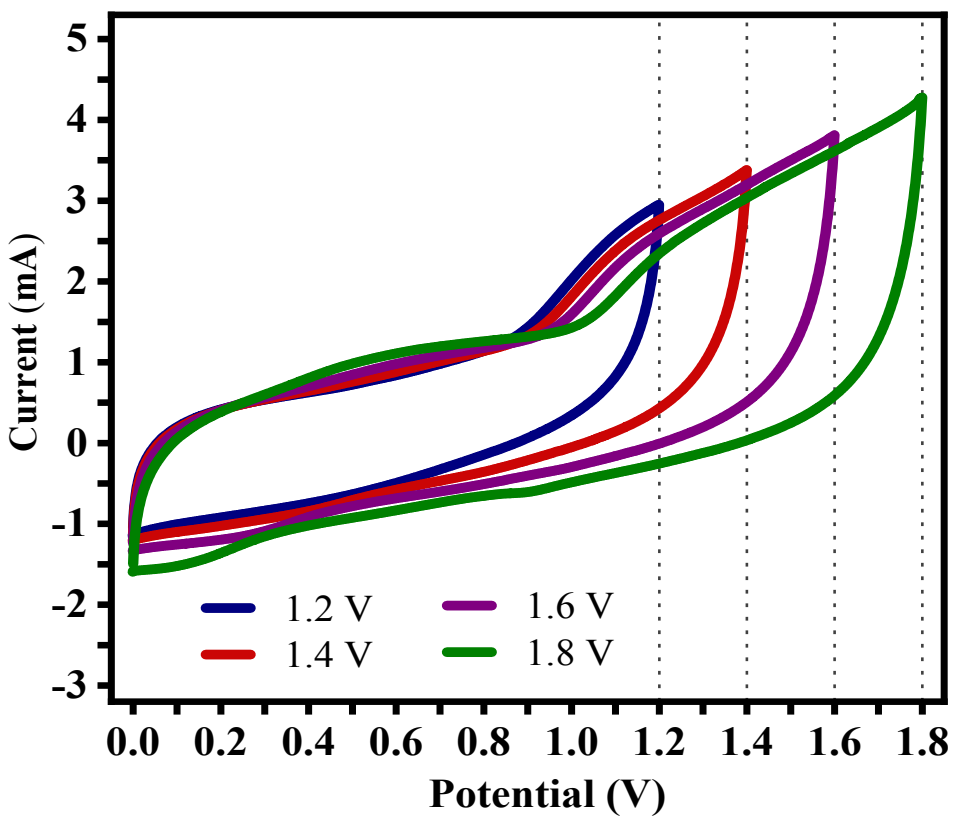

232

Figure S7 Cyclic voltammograms of the asymmetric cell (EMD/0.5 Alg and AC) with increasing the operating voltage from 1.2 to $1.8 \mathrm{~V}$ at the scan rate of $10 \mathrm{mV} \mathrm{s}^{-1}$.

\section{References}

236

(2) Cross, A.; Morel, A.; Cormie, A.; Hollenkamp, T.; Donne, S. Enhanced Manganese

(1) Sing, K. S. W. Reporting Physisorption Data for Gas/Solid Systems. Pure Appl. Chem. 1982, 54 (11), 2201-2218. Dioxide Supercapacitor Electrodes Produced by Electrodeposition. J. Power Sources 2011, 196 (18), 7847-7853.

(3) Arnott, J. B.; Williams, R. P.; Pandolfo, A. G.; Donne, S. W. Microporosity of HeatTreated Manganese Dioxide. J. Power Sources 2007, 165 (2), 581-590.

(4) Biswal, A.; Tripathy, B. C.; Li, D.; Minakshi, M. Electrodeposition of Pluronic F127 Assisted Rod-like EMD/Carbon Arrays for Efficient Energy Storage. Dalt. Trans. 2015, $44,16446-16457$.

(5) Ross Macdonald, J. Note on the Parameterization of the Constant-Phase Admittance Element. Solid State Ionics 1984, 13 (2), 147-149. 
248 (6) Schelder, W. Theory of the Frequency Dispersion of Electrode Polarization. Topology of Networks with Fractional Power Frequency Dependence. J. Phys. Chem. 1975, 79 (2), $127-136$.

251 (7) Arnott, J. B.; Donne, S. W. Examining Manganese Dioxide Graphite Connectivity in Alkaline Electrolytes. J. Electrochem. Soc. 2007, 154 (8), A776.

253

(8) Bailey, M. R.; Donne, S. W. Electrochemical Impedance Spectroscopy Study into the 254 Effect of Titanium Dioxide Added to the Alkaline Manganese Dioxide Cathode. 2011, 255 158 (7), A802-A808.

256 